\title{
THE ENVIRONMENT OF HERCULES A
}

\author{
N.A.B. GIZANI AND J.P. LEAHY \\ University of Manchester, \\ NRAL, Jodrell Bank, Cheshire, SK11 9DL, UK.
}

\section{Introduction}

The bright radio galaxy Hercules $\mathrm{A}(z=0.154)$, has power $\mathrm{P}_{178 \mathrm{MHz}}=$ $8 \times 10^{26} \mathrm{~W} \mathrm{~Hz}^{-1} \mathrm{sr}^{-1}$ and although this puts it well above the FR break, its structure is intermediate between Fanaroff and Riley classes I and II. With a linear size of $334 \mathrm{kpc}$ (for $\mathrm{H}_{0}=100 \mathrm{kms}^{-1} \mathrm{Mpc}^{-1}$ ), Hercules A possesses an unusual jet-dominated morphology and no compact hotspots. The western jet shows partial or full ring-like features that form a linear sequence heading from the core to the lobes and follow an inner jet.

The host galaxy is a $\mathrm{cD}$ at the centre of a cluster with an X-ray luminosity of $1.5 \times 10^{37} \mathrm{~W}$ (Sadun et al 1993), comparable to typical richness 0 to 1 Abell clusters. By studying the environment in detail we hope to find out whether the peculiar structure is related to the unusually dense environment.

\section{The Laing-Garrington effect}

Strongly asymmetrical Faraday depolarization is revealed by low resolution VLA polarization data (Garrington \& Holmes unpublished) at 6 and $18 \mathrm{~cm}$, the side with the stronger jet being less affected. Hence Her A exhibits a strong Laing-Garrington effect (Garrington et al 1988, Laing 1988). The depolarization seems stronger at the centre of the source, especially in the more depolarized western lobe. We are currently observing Hercules A at $\sim 1^{\prime \prime}$ resolution at several wavelengths. Preliminary results at $6 \mathrm{~cm}$ showed the polarization to be disordered in the western lobe, and mostly ordered in the eastern lobe. We interpret this disordering as the result of the Faraday rotation which causes depolarization at lower frequency and resolution. This rotation is apparently external, because at high resolution we see rotation and not depolarization. Hercules $\mathrm{A}$ is $28 \%$ polarized on average at this 
resolution, and the scale size of the rotation measure structure, and hence the external magnetic field, is $\sim 20 \mathrm{kpc}$ at the end of the western lobe, with smaller scales close to the centre. Our A-configuration observations at $L$ band show a strong depolarization of the western lobe, and a very disordered polarization in the east, rather like the western lobe at $6 \mathrm{~cm}$.

All this suggests the depolarization is caused by a centrally condensed medium in which Her A is embedded at a substantial angle. If the depolarization in the west is external, then the rotation measure is not yet resolved at L-band. Most probably the Faraday-active medium is the ' $\mathrm{X}$-ray' gas, with the asymmetry due to the weak jet and associated lobe being behind the bulk of the gas while the other lobe is in front. Therefore the jet asymmetry must be largely due to relativistic boosting.

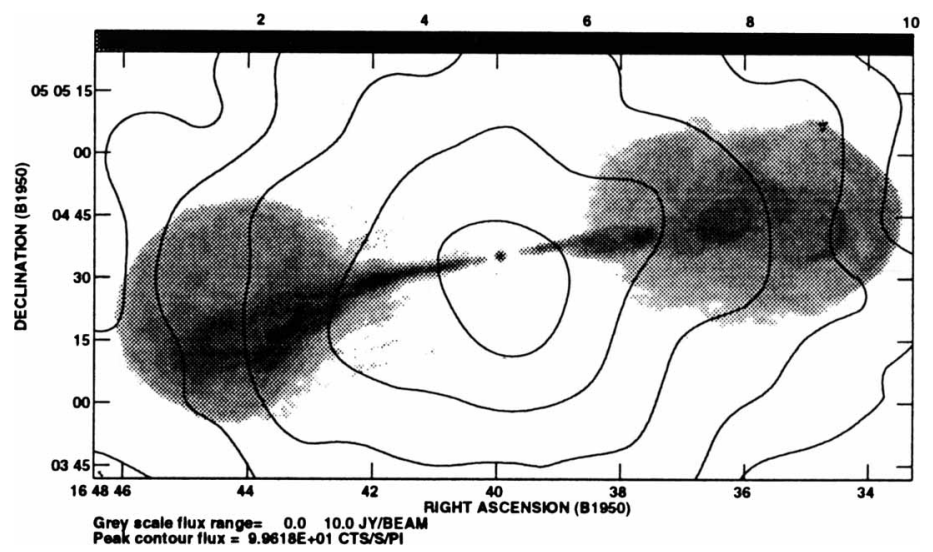

Figure 1. A contour map of the ROSAT image of Hercules A cluster in the $0.5-2 \mathrm{keV}$ band, overlaid on a grey scale of the $6 \mathrm{~cm}$ radio map. Contours are separated by a ratio of 2 .

\section{ROSAT observations}

We observed Her A with ROSAT's PSPC. The cluster emission is aligned with the radio axis and extended on roughly the same scale as the lobes. The cluster core is resolved and at most $8 \%$ of the total flux can be attributed to a nuclear component. Radial profile plots show that faint $\mathrm{X}$-ray emission extends out to $440 \mathrm{kpc}$. Global spectral fitting to the Her A cluster source in our PSPC data yields an excellent fit to a Raymond-Smith model with $\mathrm{kT} \sim 3 \mathrm{keV}$, and $\mathrm{N}(\mathrm{H})=6.2 \times 10^{20} \mathrm{~cm}^{-2}$.

\section{References}

Garrington, S.T. and Leahy, J.P. Conway, R.G. and Laing, R.A., Nat., 1984, 331, 147 Laing, R.A., Nat., 1988, 331, 149

Sadun, A.C. and Hayes, J.J.E., Astr.Soc.Pac.Publ., 1993, 105, 379 